\title{
Factores mediadores en la adquisición y desarrollo de la autodeterminación en adolescentes con discapacidad intelectual
}

\author{
Mediating factors in the acquisition and development of self-determination behavior in \\ adolescents with intellectual disabilities
}

\author{
Luisa Losada-Puente*, Manoel Baña-Castro ** \\ *Dpto. Filosofía y Métodos de Investigación en Educación, **Dpto. Psicología Evolutiva de la Educación \\ Universidade da Coruña (España).
}

\begin{abstract}
Resumen
Este trabajo analiza los resultados de varias investigaciones que estudian la mediación de los factores individuales y ambientales en la adquisición y expresión de la autodetermianción en adolescentes con discapacidad intelectual y del desarrollo. Se emplea la modalidad de investigación documental, con la revisión de veinte artículos de investigación. Los resultados evidencian la repercusión de una amplia gama de factores, así como las múltiples correlaciones entre éstos. Su estudio contribuye a mejorar la comprensión acerca de su impacto en la construcción y expresión de la autodeterminación y justifica las actuaciones diseñadas para la promoción de una plena autodeterminación.

Palabras clave: autodeterminación, discapacidad, variables predictoras, características individuales, influencias ambientales
\end{abstract}

\begin{abstract}
This paper analyses the results of several investigations that study the mediation of individual and environmental factors in the acquisition and expression of self-determination in adolescents with intellectual and developmental disabilities. A documentary research is conducted, with a review of twenty research articles. The results show the impact of a wide range of factors and multiple correlations between them. Their study contributes to better understanding of its impact on the construction and expression of self-determination and justifies actions designed to promote full self-determination.

Keywords self determination, disabilities, predictor variables, individual characteristics, environmental influences.
\end{abstract}

En las últimas décadas, el principio de autodeterminación ha comenzado a erigirse en el terreno científico y profesional como referente en la la provisión de servicios para personas con discapacidad intelectual y otras alteraciones del desarrollo, y en un estándar en la lucha contra la opresión social gestada desde los medios de comunicación, las fundaciones benéficas, los profesionales de diferentes ámbitos, la política en general o las formas de producción, así como para la defensa de la capacidad de decidir y opinar de estas personas (Walker et al., 2011). Junto a otros conceptos como la emancipación, los derechos y la dignidad, la autoestima, la eficacia y la autoeficacia, percepción de la valía y calidad de vida, la autodeterminación se ha ido introduciendo de forma progresiva en la investigación y en los servicios que se ofrecen a las personas con discapacidad.

La atención prioritaria de los profesionales y servicios al estudio de la necesidades de apoyo de las persona con discapacidad con el fin de proveerlas de los medios y recursos necesarios para alcanzar los resultados personales deseados y la mejora de su calidad de vida, junto con el creciente número de publicaciones que hemos encontrado que versan sobre la autodeterminación y sus repercusiones sobre la CDV de estas personas, justifican el interés de este estudio por mejorar la comprensión de este amplio y complejo concepto analizando la repercusión de las características personales y el entorno en que se desarrolla el individuo sobre la vida de los adolescentes con discapacidad intelectual y del desarrollo.

Los avances de los últimos años en la conceptualización de este constructo permiten afirmar, hoy en día, la confluencia de múltiples factores y variables sobre la consecución de una mayor autodeterminación en las diversas etapas de la vida. En la actualidad, se reconoce que la autodeterminación no puede ser explicada únicamente por la influencia de las características biológicas, genéticas o personales, ni tampoco por el impulso de factores externos, leyes naturales o fuerzas ajenas a la persona, sino que resulta de la confluencia de múltiples factores y conceptos que predicen o anteceden $\mathrm{y}$, por tanto, causan la autodeterminación o surgen como efecto de su manifestación por parte de las personas con discapacidad intelectual y otras alteraciones del desarrollo.

Esta asunción ha llevado a numerosos investigadores e investigadoras a analizar, a nivel teórico y empírico, el papel mediador de los diversos factores personales y ambientales sobre la promoción de la autodeterminación (véase Tabla 1). 
Tabla 1.

Factores mediadores de la autodeterminación

\begin{tabular}{|c|c|c|}
\hline & & Variables \\
\hline \multirow{5}{*}{$\begin{array}{l}\text { Factores } \\
\text { internos }\end{array}$} & & Edad \\
\hline & Biológicos y & Género \\
\hline & & Funcionamiento intelectual \\
\hline & Conductuales & Conducta adaptativa \\
\hline & personalidad & Valores, creencias y actitudes \\
\hline \multirow{9}{*}{$\begin{array}{l}\text { Factores } \\
\text { externos }\end{array}$} & \multirow{3}{*}{ Contextuales } & Entorno próximo (vivienda) \\
\hline & & Cultura/ étnia \\
\hline & & Entorno escolar/laboral \\
\hline & \multirow{4}{*}{ Familiares } & Estatus socioeconómico \\
\hline & & Estilos de educación parental \\
\hline & & $\begin{array}{l}\text { Conocimiento, valores } \quad \mathrm{y} \\
\text { actitudes familiares }\end{array}$ \\
\hline & & Escolarización (tipo de centro) \\
\hline & \multirow[t]{2}{*}{ Escolares } & $\begin{array}{l}\text { Prácticas pedagógicas y } \\
\text { actitudes del profesorado }\end{array}$ \\
\hline & & $\begin{array}{l}\text { Posibilidades y deseo de } \\
\text { participación }\end{array}$ \\
\hline
\end{tabular}

La asunción de los presupuestos de la teoría de la agencia causal de toda conducta autodeteterminada (Wehmeyer, 2013) implica considerar que cada acto causado por la persona se produce de forma volitiva y mediada por las características o factores internos de ésta. Concretamente, la literatura científica actual se refiere a la repercusión de variables como la inteligencia, la edad, el género, las habilidades sociales y la conducta adaptativa o los valores, creencias y actitudes de la persona sobre la promoción de la autodeterminación en la persona (véase Tabla 1). Del mismo modo, algunas investigaciones han prestado especial atención al influjo del ambiente global y próximo que rodea a la persona sobre la consecución de una conducta autodeterminada (Stancliffe, Abery y Smith, 2000; Wehmeyer, Abery, Mithaug y Stancliffe., 2003).

Por ello, este estudio pretende contribuir a la investigación actual que versa sobre la mediación de las características individuales y del ambiente sobre la adquisición y desarrollo de la conducta autodeterminada. De este modo, se plantea como objetivo analizar las principales investigaciones desarrolladas en los últimos años, acerca de la influencia de variables individuales y ambientales en la conducta autodeterminada de las personas con discapacidad intelectual y otras alteraciones del desarrollo, atendiendo especialmente a la etapa de la adolescencia y la transición a la vida adulta.

\section{Método}

El estudio emplea la modalidad de investigación documental encuadrada dentro de la metodología de tipo cualitativo. Con esta modalidad de estudio se posibilita el regreso al pensamiento original de los estudiosos sobre el tema, ofreciendo así mayor rigor a las fuentes originales mediante la exploración de diversas e interconectadas interpretaciones de un mismo suceso (Gómez, 2011; Hodder, 2012).

\section{Materiales}

El material escrito empleado en esta investigación son las fuentes primarias y secundarias que registran información acerca de la mediación de los factores individuales y ambientales sobre la autodeterminación, extraídos de seis bases de datos nacionales e internacionales de las áreas de educación y psicología: CSIC ISOC - Ciencias Sociales y Humanidades; DIALNET; ERIC; PsycINFO; SCOPUS; Web Of Science, WOS.

\section{Procedimiento}

La búsqueda en bases de datos se desarrolla introduciendo uno o varios de los siguientes términos en español y en inglés: autodeterminación (o self-detemination), factores (o factors); factores ambientales (o environmental factors) y discapacidad (o disability), con la que fueron extraídos más de doscientos documentos. Éstos fueron filtrados empleando descriptores relacionados con los conceptos de autodeterminación, discapacidad, educación especial, dificultades de aprendizaje, ambiente, características individuales, edad, funcionamiento intelectual, conducta adaptativa, habilidades sociales, actitud del estudiante, entorno próximo, vivienda, educación especial, familias, etc.

Se eliminan los artículos con referencias a la autodeterminación como derecho político o colectivo, en relación con la salud y con el deporte, aquellos que no se referían a la autodeterminación como constructo global, y las revisiones teóricas cuyo foco de atención no fuesen los factores que afectan al desarrollo de la conducta autodeterminada. Se emplean los filtros de las bases de datos para excluir documentos referentes a áreas de economía y empresa, medicina, bioquímica y biología genética y molecular y enfermería. Finalmente, se introduce un filtro temporal, eliminando aquellas investigaciones anteriores al año 2000.

La selección de textos da como resultado un total de veinte documentos de los últimos quince años, seleccionados por interés y relevancia de sus títulos y resúmenes en los que se recogen las aportaciones que evidencian la influencia de las características personales (edad, sexo, funcionamiento intelectual) así como desde los entornos más globales, cuya influencia afecta de modo menos directo a la persona (la cultura) hasta aquellos que repercuten directamente sobre la persona, relacionados con las características del entorno en que se desenvuelve la persona, con el estatus socioeconómico y los estilos de educación familiar, la escolarización y la participación en el proceso de transición a la vida adulta.

\section{Resultados}

El análisis documental ha permitido atender a los principales factores individuales y ambientales considerados mediadores o predictores de la autodeterminación en personas con discapacidad intelectual y del desarrollo. A continuación 
presentaremos las principales consideraciones de algunos de los factores que afectan a la adquisición y expresión de la autodeterminación. Concretamente, analizaremos el papel del funcionamiento intelectual, la edad, el género, la cultura, la familia y la escuela.

\section{Factores individuales}

Nivel de inteligencia o funcionamiento intelectual. Las primeras investigaciones en el campo destacan la influencia del coeficiente intelectual (CI) sobre la adquisición de la conducta autodeterminada en personas con discapacidad intelectual entendiendo, por tanto, que sus limitaciones en el número y la complejidad de habilidades suponen un freno a su capacidad para actuar como agente causal de su vida. Desde hace décadas, se reconoce que la dificultad cognitiva no está reñida con la capacidad de la persona para alcanzar la plena autodeterminación (Bissoto, 2014; Wehmeyer, 2006a; Wehmeyer et al., 2011). Son múltiples los estudios que analizan la relación entre autodeterminación y CI corroborando, en su mayoría, la existencia de una fuerte correlación entre ambos, pero matizando que la inteligencia no tiene por que ser un factor determinante ni predictor de la conducta autodeterminada (Chambers et al., 2007; Gómez-Vela, Verdugo, González-Gil, Badía y Wehmeyer, 2010; Gómez-Vela, Verdugo, González-Gil, Badía y Wehmeyer, 2012; Lee et al., 2012; Vega García, Gómez Vela, Fernández Pulido y Badía Corbella, 2013; Wehmeyer y Gardner, 2003).

Edad. La investigación actual evidencia que la adquisición de las habilidades conducentes a la expresión de la autodeterminación es un proceso que se sucede en un continnum a lo largo de la vida, por medio de instrucción directa y cuyo punto álgido se produce en la adolescencia, momento en el que la persona comienza a emplear sus aprendizajes en la práctica de su vida diaria (Bissoto, 2014; Gómez-Vela et al., 2010; Peralta, como se citó en Vega García et al., 2013; Wehmeyer, 2013). Se ha constatado la estrecha relación entre mayor autodeterminación y mejores resultados en la adultez para la juventud con discapacidad así como mayores problemas de inadaptación, aislamiento, cambios de humor y problemas comunicativos en la adultez, en jóvenes con menores niveles de autodeterminación (Nota et al., 2007; Shogren, Wehmeyer, Palmer y Paek, 2013; Vega García et al., 2013; Wehmeyer, 1996; Wehmeyer y Palmer, como se citó en Nota et al., 2007).

Género. Los presupuestos del enfoque centrado en la igualdad de género postulan que, más allá del desarrollo de las características sexuales relacionadas con la pubertad, no deben existir diferencias entre hombres y mujeres en cuanto a la consecución de un mayor nivel de autodeterminación. Lo cierto es que no existen, todavía, datos concluyentes acerca de tales diferencias (Wehmeyer, 2006a; Wehmeyer et al., 2011), pues la información es limitada y, en su mayoría, contradictoria. Ejemplos de tales discordancias se pueden observar en los resultados que nos ofrecen los estudios de Gómez-Vela et al. (2012), Lee et al. (2012), Nota et al. (2007), Soresi, Ferrara y Nota (como se citó en Shogren, 2007), Shogren et al. (2013) o Wehmeyer y Gardner (2003).

\section{Factores ambientales}

Cultura. Desde la Teoría de la Autodeterminación se asume que las necesidades a nivel competencial, de autonomía y de interacción social son innatas, universales y compatibles y su cumplimiento favorece el correcto funcionamiento de todos los individuos en las diversas culturas y sociedades (Hui y Tsang, 2012). Ahora bien, pese a la universalidad de este constructo, su aprendizaje y expresión así como la importancia y utilidad conferida por cada individuo puede variar en función de la cultura de pertenencia (Shogren et al., 2007). La cultura de cada lugar, como comportamiento aprendido y compartido, modela las percepciones, las formas de comportamiento y el sentido de la realidad de cada persona (Wehmeyer et al., 2011). El interés por indagar sobre la influencia del factor cultural sobre la expresión de la autodeterminación es todavía incipiente, encontrando tan solo un limitado número de publicacines que demuestran la existencia de diferencias en los niveles de autodeterminación en función de la cultura (Frankland et al. como se cita en Wehmeyer et al., 2011; Leake y Boone, 2007; Trainor, 2005; Zhang, 2005; Zhang, Landmark, Grenwelge y Montoya, 2010) con resultados muy dispares y poco concluyentes.

Familia. La mayor parte de la investigación sobre la influencia de la familia en la conducta autodeterminada de los hijos e hijas ha puesto su atención en el análisis del estatus socioeconómico y los estilos educativos parentales revelando la existencia de una compleja red de relaciones entre estatus, educación parental y autodeterminación (Leake y Boone, 2007; Trainor, 2005; Wehmeyer et al., 2011; Zhang, 2005). Así mismo, se ha indagado sobre las percepciones, creencias y actitudes familiares hacia la promoción de la autodeterminación de sus hijos/as con discapacidad intelectual. Los estudios de Carter et al. (2009) y Chambers et al. (2007) han revelado la influencia de las percepciones y creencias familiares como barreras o facilitadores a la adquisición y expresión de habilidades relacionadas con la autodeterminación en los hijos/as y, por su parte, Zhang (2005) evidencia el impacto que tiene la predisposición de los padres a que sus hijos/as decidan sobre aspectos importantes en sus vidas.

Escuela. Diversas investigaciones encuentran niveles superiores en autodeterminación y mayor autonomía y conocimiento sobre si mismos en el alumnado con discapacidad intelectual escolarizado en centros específicos (Shogren et al., 2013; Vega García et al., 2013) o integrado en aulas específicas (Cho, Wehmeyer y Kingston, 2012; Zhang, como se cita en Shogran et al., 2013). Respecto a las prácticas pedagógicas, una buena parte del profesorado comprende la importancia de la autodeterminación como un aspecto esencial en el proceso educativo de todo el alumnado (Cho et al., 2012; Gómez-Vela et al., 2012), destacando sus beneficios para la consecución de una mayor inserción y mejor calidad de vida. No obstante, esta positiva percepción del profesorado acerca de las posibilidades de sus estudiantes varía en función del grado de discapacidad de cada alumno, percibiéndose distorsionado el trato recibido por el alumnado con discapacidades más graves 
o severas por parte del profesorado. El profesorado que trabaja con alumnado de discapacidad leve es más proclive a considerar importante la formación del alumnado en autodeterminación y su propia formación e información sobre cómo fomentar la conducta autodeterminada en su alumnado, que aquellos que trabajan con alumnado de discapacidad severa (Cho et al., 2012; Shogren et al., 2013; Wehmeyer, 2006b).

Y en relación con las posibilidades que se le ofrece al alumnado para involucrarse en la vida escolar, se evidencia la importancia de la participación del alumnado en el desarrollo de la autodeterminación y, si bien es confuso el carácter predictor de este factor (Shogren et al., 2013; Shogren et al., 2007), se ha demostrado su relación con la obtención de resultados positivos en la adultez (Wehmeyer et al., 2008; Wehmeyer et al., 2010).

\section{Discusión}

Este trabajo se ha centrado en analizar algunos de los principales factores involucrados en la adquisición y expresión de las habilidades relacionadas con la conducta autodeterminada.

Los estudios centrados en las características individuales evidencian la relación entre funcionamiento intelectual y autodeterminación aunque su carácter predictor ha quedado en entredicho. En relación al género, los resultados continúan siendo poco concluyentes. Y en relación con la edad, queda demostrada la incidencia de este factor así como la importancia de estimular desde tempranas edades las habilidades conducentes al desarrollo de un comportamiento autodeterminado.

Por su parte, los estudios que versan con los factores ambientales mediadores de la conducta autodeterminada concluyen la influencia de un entorno estimulante que favorezca la adquisición y expresión de comportamientos autodeterminados así como la relevancia que tienen las pautas educativas parentales y la percepción de los padres y madres acerca de las capacidades de sus hijos. Igualmente, se evidencia la correlación entre el tipo de prácticas pedagógicas y actitudes del profesorado y la consecución de mayores niveles de autodeterminación, al igual que ocurre con las oportunidades que se les ofrezcan a estos jóvenes de experimentar en situaciones naturales, de poner a prueba sus capacidades, de reconocer sus limitaciones y de equivocarse y acertar.

El estudio de estos factores contribuye, por un lado, a ampliar la comprensión acerca de su impacto sobre la construcción y la expresión de la conducta autodeterminada $\mathrm{y}$, por otro lado, a justificar las actuaciones que se enfocan hacia la promoción de la plena autodeterminación de las personas.

No obstante, pese a los avances de las últimas décadas en materia de autodeterminación, continúan existiendo ciertas lagunas en el estudio de los factores subyacentes al desarrollo y expresión de la autodeterminación, sobre todo, en la etapa de la adolescencia.

Por tanto, se hace necesario continuar la investigación en esta línea, de forma que contribuya a la mejor comprensión de los elementos que afectan al desarrollo de la conducta autodeterminada en cada etapa del desarrollo y que nos permitan facilitar prácticas que favorezcan su adquisición en personas con discapacidad intelectual

\section{Referencias}

Bissoto, M.L. (2014). Deficiência intelectual e processos de tomada de decisão: estamos enfrentando o desafio de educar para a autonomia?. Educação Unisinos, 18(1), 3-12. doi.: 10.4013/edu.2014.181.01

Carter, E.W., Trainor, A., Owens, L., Sweden, B. y Sun, Y. (2009). Self-determination prospects of youth with high-incidence disabilities: divergent perspectives and related factors. Journal of Emotional and Behavioral Disorders, 18(2), 67-81. doi.: 10.1177/1063426609332605

Chambers, C.R., Wehmeyer, M.L., Saito, Y., Lida, K.M., Lee, Y. y Singh, V. (2007). Self-determination: What do we know? Where do we go?. Exceptionally, 15(1), 3-15. doi.: 10.1080/09362830709336922

Cho, H.-J., Wehmeyer, M.L. y Kingston, N.M. (2012). The effect of social and classroom ecological factors on promoting self-determination in elementary school. Preventing School Failure: Alternative Education for Children and Youth, 56(1), 19-28. doi.: 10.1080/1045988X.2010.54419

Gómez, L.E. (2011). Un espacio para la investigación documental. Revista Vanguardia Psicológica, 1(2), 226-233. http://umb.edu.co:82/revp/index.php/vangua rdiapsicologica/article/viewArticle/36

Gómez-Vela, M., Verdugo, M.A., González-Gil, F., Badía, M. y Wehmeyer, M.L. (2012). Assessment of self-determination of Spanish students with intellectual and other educational needs. Journal of Applied Research in Intellectual Disabilities, 23(5), 505-506. www.ncbi.nlm.nih.gov/pmc/articles/PMC41 04407/

Hodder, I. (2012). The interpretation od documents and material culture. En J. Goodwin (Ed.), Sage biographical research (pp. 171-188) (Vol. 1-4) (5 ed.) [versión Adobe Digital Editions]. doi.: 10.4135/9781446268537

Hui, E.K.P. y Tsang, S.K. (2012). Self-determination as a psychological and positive youth development construct. The Scientific World Journal, 2012, 1-7. doi.: 10.1100/2012/759358

Leake, D. y Boone, R. (2007). Multicultural perspectives on self-determination from youth, parent, and teacher focus group. Career Development for Exceptional Individuals, 30(2), 104-115. Doi.: 10.1177/08857288070300020101

Lee, Y., Wehmeyer, M, Palmer, S., William-Diehm, K., Davies, D. y Stock, S. (2012). Examining individual and instruction-related predictors of the self-determination of students with disabilities: multiple regression analysis. Remedial Special Education, 33(3), 150-161. doi.: 10.1177/0741932510392053

Nota, I., Ferrari, L., Soresi, S. y Wehmeyer, M.L. (2007). Self-determination, social abilities and the quality of 
life of people with intellectual disabilities. Journal of Intellectual Disability Research, 51(11), 850-865. doi.: 10.1111/j.1365-2788.2006.00939.x

Peralta, F. (2007). La autodeterminación de las personas con discapacidad intelectual como meta educativa. En M. Liesa, P. Allueva y M. Puyuelo (Coords.), Educación y acceso a la vida adulta en personas con discapacidad (pp.263-277). Barbasto: Fundación Ramón J. Sender.

Rojas Pernia, S. (2004). Autodeterminación y calidad de vida en personas discapacitadas. Experiencia desde un hogar de grupo (Tesis doctoral). http://www.tdx.cat/bitstream/handle/10803/ 5044/srp1de2.pdf?sequence $=1$

Shogren, K.A., Wehmeyer, M.L., Palmer, S.B. y Paek, Y. (2013). Exploring personal and school environment characteristics that predicts self-determination. Exceptionality: a Special Education Journal, 21(3). doi.: 10.1080/09362835.2013.802231

Shogren, K.A., Wehmeyer, M.L., Palmer, S.B., Soukup, J.H., Little, T.D., Garner, N. y Lawrence, M. (2007). Examining individual and ecological predictors of the self-determination of students with disabilities. Exceptional Childen, 73(4), 488-509. eirc.ed.gov/?id=EJ817517

Stancliffe, R., Abery, B. y Smith, J. (2000). Personal control and the ecology of community living settings: beyond living-unit size and type. Mental Retardation, 100, 59-67. doi.: 10.1352/0895-8017.2000.105

Trainor, A. (2005). Self-determination perceptions and behaviors of diverse students with LD during the transition planning process. Journal of Learning Disabilities, 38(3), 233-248.

Vega García, C., Gómez Vela, M., Fernández Pulido, R. y Badía Corbella, M. (2013). El papel del contexto educativo en la autodeterminación. Análisis de su influencia en el proceso de transición a la vida adulta de alumnos con discapacidad intelectual. Revista Iberoamericana de Educación, 63, 19-33. http://riberdis.cedd.net/bitstream/handle/1118 1/4359/El_papel_del_contexto_educativo.pdf?sequen $\mathrm{ce}=1 \& \mathrm{rd}=0031371311959578$

Walker, H.M., Calkins, C., Wehmeyer, M.L., Walker, L., Bacon, A., Palmer, S.B.,... Jonhson, D.R. (2011). A social-ecological approach to promote self-determination. Exceptionaly: A Special Education Journal, 19(1), 6-18. doi.: 10.1080/09362835.2011.537220

Wehmeyer, M.L. (1996). Self-determination as an educational outcome: Why is it important to children, youth and adults with disabilities?. En D.J. Sands y M.L. Wehmeyer (Eds.), Self-determination across the life span: independence and choice for people with disabilities (pp. 15-34). Baltimore: Paul H. Brookes.

(2006a). Autodeterminación y personas con discapacidades severas. Siglo Cero, $37(4)$,

5-16. http://www.feaps.org/comunicacion/documento s/siglo_220.pdf

. (2006b). Factores intraindividuales y medioambientales que afectan a la autodeterminación. $\begin{array}{lll}\text { Siglo Cero, } & \text { 37(4), }\end{array}$
47-56. http://sid.usal.es/idocs/F8/ART9355/articulos4. pdf

(2013). Self-determination. En Autor

(Coord.), The Oxford handbook of positive psychology and disability (pp. 116-136). Nueva York, EE.UU.: Oxford University Press.

Wehmeyer, M.L., Abery, B., Mithaug, D.E. y Stancliffe, R.J. (2003). Theory in self-determination: foundations for educational practice. Springfield, IL: Charles C Thomas Publisher, LTD.

Wehmeyer, M.L. y Gardner, N.W. (2003). The impact of personal characteristics of people with intellectual and developmental disability on self-determination and autonomus functioning. Journal of Applied Reseach in Intellectual Disabilities, 16, 255-265. doi.: 10.1046/j.1468-3148.2003.00161.x

Wehmeyer, M.L., Palmer, S.B., Shogren, K., Williams-Diehm, K. y Soukup, J.H. (2010). Establishing a causal relationship between intervention to promote self-determination and enhanced student self-determination. Journal of Special Education, 46(4), 195-210. doi.: 10.1177/0022466910392377

Wehmeyer, M.L., Abery, B.H., Zhang, D., Ward, K., Willis, D., Hossain Amin, W., ... Walker, H. (2011). Personal self-determination and moderating variables that impact efforts to promote self-determination. Exceptionally, 19(1), 19-30. www.aucd.org/docs/ScalingUpPaper2Final.pd $\mathrm{f}$

Wehmeyer, M.L., Palmer, S.B., Soukup, J.H., Garner, N.W. y Lawrence, M. (2008). Self-determination and student transition planning knowledge and skills: predicting involvement. Exceptionally: A Special Education Journal, 15(1), 31-44. doi.: 10.1080/09362830709336924

Zhang (2005). Parent practices in facilitating self-determination skill: the influences of cultura, socioeconomic status, and children's special education status. Research and Practice for Persons with Severe Disabilities, 20(2), 154-162. doi.: 10.2511/rpsd30.3.154

Zhang, D., Landmark, L., Grenwelge, C. y Montoya, L. (2010). Culturally diverse parents' perspective on self-determination. Education and Training in Autism and Developmental Disabilities, 45(2), 175-186. http://eric.ed.gov/?id=EJ884372 